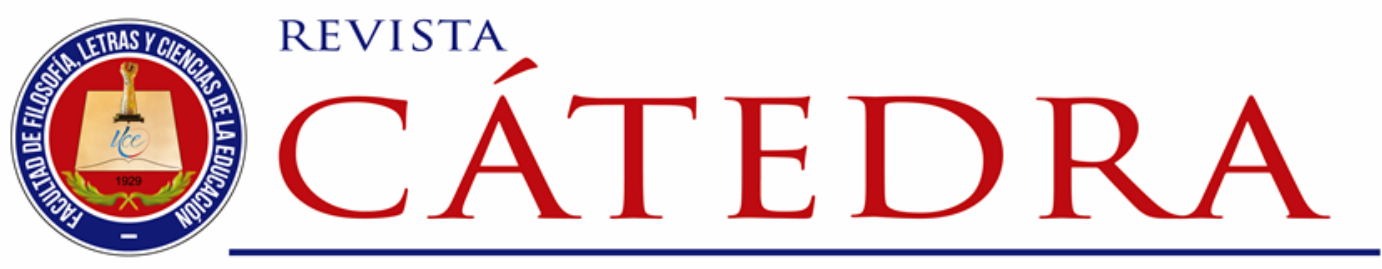

\title{
La universidad intercultural mexiquense y las comunidades rurales e indígenas. Estrategias de acercamiento y experiencias de vinculación
}

\section{The intercultural mexiquense university and rural and indigenous communities. Strategies of rapprochement and linkage experiences}

\author{
Zuzana Erdösová \\ Universidad Autónoma del Estado de México, México \\ zerdosova@uaemex.mx \\ https://orcid.org/0000-0001-7924-7599
}

(Recibido: 01/03/2021; Aceptado: 15/03/2021; Versión final recibida: 05/05/2021)

Cita del artículo: Erdösová, Z. (2021). La universidad intercultural mexiquense y las comunidades rurales e indígenas. Estrategias de acercamiento y experiencias de vinculación. Revista Cátedra, 4(2), 70-87.

\section{Resumen}

El modelo educativo de la universidad intercultural tiene como objetivo la construcción del conocimiento en condiciones de equidad y diálogo con los pueblos originarios, sin embargo, debido a la carga ideológica neoindigenista del modelo educativo, el enfoque teórico y las prácticas reales suelen discrepar. Por ello, el artículo analiza los modos de relacionarse de una universidad concreta, la Universidad Intercultural del Estado de México, con las comunidades indígenas de la región. Se realiza dicho análisis a través de los testimonios de una serie de protagonistas indígenas y no indígenas, involucrados en los procesos de la Vinculación Comunitaria, uno de los ejes del modelo educativo. Se concluye que la relación universidad-comunidad se caracteriza, por un lado, por la apertura de la Universidad a las iniciativas surgidas desde las comunidades incluyendo a los alumnos que de ellas proceden, pero, por otro, por una actitud modernizante que da prioridad a las nociones del desarrollo económico entendido en términos neoliberales ante la autonomía cultural del desarrollo propia de los pueblos originarios. En este sentido, ir desmitificando los discursos educativos hegemónicas se hace de primera importancia para asegurar la transparencia del sistema educativo y su funcionalidad en el contexto de diversidad étnica. 


\section{Palabras clave}

Comunidad, interculturalidad, pueblos indígenas, universidad mexicana, vinculación.

\section{Abstract}

The educational model of the intercultural university aims at the construction of knowledge in conditions of equity and dialogue with indigenous peoples, however, due to the neoindigenist ideological load of the educational model, the theoretical approach and actual practices often diverge. For this reason, the article analyzes the ways in which a specific university, the Intercultural University of the State of Mexico, relates to the indigenous communities of the region. This analysis is carried out through the testimonies of a series of indigenous and non-indigenous protagonists, involved in the processes of Community Liaison, one of the axes of the educational model. It is concluded that the universitycommunity relationship is characterized, on the one hand, by the openness of the University to the initiatives arising from the communities, including the students who come from them, but, on the other hand, by a modernizing attitude that gives priority to the notions of economic development understood in neoliberal terms before the cultural autonomy of the development of the native peoples. In this sense, demystifying the hegemonic educational discourses becomes of primary importance to ensure the transparency of the educational system and its functionality in the context of ethnic diversity.

\section{Keywords}

Community, interculturality, indigenous peoples, mexican university, linkage.

\section{Introducción}

La problemática de la educación superior intercultural en América Latina es un tema emergente e insuficientemente conocido aún. Llama la atención de los investigadores por su alta relevancia social, ya que se relaciona con algunas de las problemáticas más sensibles del mundo globalizado, tales como: la exclusión social, pobreza, relaciones interculturales o derechos culturales y colectivos, relación entre las minorías y la mayoría, etc. Ante tal contexto complejo, es importante resaltar que, en Latinoamérica, las llamadas universidades interculturales y/o indígenas distan de ser un fenómeno educativo homogéneo; en realidad se trata de una serie de modelos educativos que han ido emergiendo en el continente aproximadamente desde la década de los años 90. De alguna manera, todos estos modelos se posicionan contra las políticas educativas que intentan simplemente incluir a los jóvenes de los grupos étnicos en las estructuras universitarias existentes. En síntesis, dichos modelos educativos se caracterizan por lo siguiente:

Primero, ofrecen un tipo de educación superior cuya concepción y modalidad puede variar de manera significativa (formal, más bien informal, itinerante...). Segundo, manejan un concepto de la interculturalidad, el que presenta una inmensa variación semántica y está sometido a una gama de manejos discursivos que dependen de los objetivos buscados. En el centro de atención está la relación entre el estado-nación representado por la sociedad mayoritaria y los diferentes grupos étnicos vistos como discrepantes de este modelo dominante.

Tercero, en su seno generalmente se forman tanto los miembros de las sociedades mayoritarias como los de los grupos étnicos tradicionalmente vistos como marginados por estas sociedades. Sin embargo, la población meta a la que se dirigen estas políticas 
educativas, suele ser claramente la étnica. Cuarto, mantienen diferentes tipos de relaciones con las instancias oficiales de los países en que se sitúan (por ejemplo, hay universidades interculturales financiadas por el estado y otras económicamente independientes). De acuerdo con ello, las universidades interculturales pueden tener diferentes gestores (gobiernos, movimientos indígenas, etc.), circunstancia que preestablece qué tipo de relación epistémica sostendrá cada modelo educativo con la ideología pedagógica moderna y globalizada. De manera que las universidades interculturales se sitúan en diferentes puntos del eje delimitado en un extremo por las posturas claramente decoloniales y epistemológicamente alternativas a la tradición intelectual occidental, y en el otro extremo por lo que podríamos llamar el neoindigenismo ${ }^{1}$.

Si nos detenemos en el primer caso mencionado, podemos afirmar que las universidades interculturales surgen en la periferia de las sociedades latinoamericanas, impulsadas por los pueblos indígenas mismos, y como tales incluyen en sus proyectos el replanteamiento de las relaciones sociales existentes, a menudo ligadas a las demandas autonómicas y los modos de vida que desprenden de las cosmovisiones originarias (por ejemplo, la corriente del Buen Vivir conocida como Sumak Kawsay o Suma Kamaña).

En cambio, en el segundo caso, las universidades interculturales impulsadas por los gobiernos nacionales son entendidas como una especie de acción afirmativa (discriminación positiva) que debe compensar la tradicional inequidad en el acceso de los individuos indígenas a la educación superior. Suelen operar con el concepto de la "pertinencia educativa" que equivale a la adecuación curricular con las realidades culturales de los pueblos que las universidades interculturales atienden.

México es indudablemente el país que cuenta con más universidades interculturales de toda América Latina. En el marco del Plan Nacional de Educación (PNE) elaborado por el gobierno de Vicente Fox (2000-2006) se delineó la fundación de diez universidades de este tipo, número que incluso ha sido rebasado. La mayoría de ellas fue fundada desde las instancias federales y todas reciben financiamiento combinado federal y estatal.

Ahora bien, el modelo educativo de la universidad intercultural mexicana se enmarca en la categoría neoindigenista anteriormente mencionada, por las siguientes razones².

1) Es un instrumento del estado. Pertenece al Subsistema de la Educación Superior del Sistema Educativo Nacional, y es gestionado por el organismo de la Secretaría de Educación Pública (SEP) llamado Coordinación General de la Educación Intercultural Bilingüe (CGEIB).

2) Es una concepción educativa creada desde fuera de los pueblos originarios. En el modelo educativo, los pueblos originarios figuran como "entes atendidos", sin tener palabra decisiva en la conceptualización de la educación intercultural que se les

\footnotetext{
${ }^{1}$ En la actualidad, la ideología indigenista mexicana del inicio del siglo XX (extendida por el resto de la región latinoamericana) se perpetúa en forma de un "neoindigenismo". El neoindigenismo mantiene la actitud paternalista y asistencialista que lo caracterizaban en sus orígenes, aunque en la actualidad tiende evitar hablar de la asimilación cultural directa, sino que maneja una retórica más sofisticada del "desarrollo de los pueblos indígenas" vistos como entes marginados y no participantes en los beneficios de la civilización moderna (Korsbaek y Sámano, 2007).

2 La información que sigue deriva de la lectura crítica y análisis discursivo del manual del modelo educativo en cuestión. Para información más detallada, véase: Erdösová y Juárez, 2017; Erdösová, 2015; Erdösová, 2013.
} 
brinda, y sin participar intelectual y activamente en la gestión práctica de las universidades interculturales respectivas.

3) Practica un "diálogo de saberes" unilateral. El concepto del diálogo de saberes que justifica la existencia de las universidades interculturales prioriza la concepción moderna occidental del ser (éxito, competencia, competitividad, desarrollo, crecimiento, progreso, innovación, noción econocéntrica del bienestar). En extensión, el modelo educativo ofrece una formación basada en la estructura pedagógica convencional (aulas, profesores, exámenes de conocimiento, excelencia educativa, formación por competencias) sin otorgar valor a los mecanismos tradicionales de la educación practicada en las comunidades indígenas.

4) Busca una interculturalidad idealizada. El concepto de la interculturalidad que maneja el modelo educativo, deja de lado las desigualdades socioculturales y el desequilibrio entre poderes entre los actores involucrados en el proyecto. De tal modo que se limita a postular lo deseable de una sociedad armónica de diálogo y cooperación entre las culturas que conforman México; las mismas que se perciben como bloques separados en necesidad de establecer comunicación. Asimismo, al priorizar el desarrollo (sobre todo económico) de las zonas atendidas, las universidades interculturales relegan al segundo plano la urgencia de una interculturalidad multilateral que atraviese la sociedad mexicana entera.

5) Practica acciones afirmativas. A pesar de admitir tanto a los alumnos indígenas como a los no indígenas, el énfasis del modelo educativo está puesto en los primeros.

Derivado de las consideraciones anteriores, en este texto establecimos el objetivo de analizar y explicar cómo se relacionan los profesionistas formados por las Universidades Interculturales con los ámbitos comunitarios a través de una estrategia intercultural denominada Vinculación con la Comunidad o Vinculación Comunitaria. Ésta representa uno de los ejes principales de la teoría educativa respectiva y forma parte inseparable tanto de los procesos de investigación, como de los de enseñanza y aprendizaje. Cabe destacar que impactar sobre la sociedad local es el macroobjetivo del modelo educativo. La universidad intercultural pone al centro el vínculo con la sociedad, "pero no de la sociedad en abstracto, sino de la sociedad directa" (Bastida y Albino, 2011, pág. 327). Para este fin, la universidad intercultural dispone del mecanismo de la Vinculación con la Comunidad, la que se determina en la teoría educativa de la siguiente manera:

Se plantea la vinculación con la comunidad como un conjunto de
actividades que implica la planeación, organización, operación y
evaluación de acciones en que la docencia y la investigación se relacionan
internamente en el ámbito universitario y externamente con las
comunidades para la atención de problemáticas y necesidades
específicas. (...) Las acciones de vinculación con la comunidad coadyuvan
al desarrollo social, cultural y productivo de las comunidades, orientando
no sólo el quehacer formativo sino también las líneas de investigación
derivadas de la realidad a la que se aportarán soluciones. Así, la
Universidad Intercultural cumple su función de pertinencia académica y
social al contribuir a la solución de problemas de su entorno y establecer
una relación de beneficio mutuo entre ella y la comunidad (Casillas y
Santini, 2006, pág. 153).

En otras palabras, la finalidad de la Vinculación Comunitaria es convertir al alumno en un protagonista activo de su propia realidad a través del trabajo de campo realizado en los ámbitos comunitarios. De esta manera se considera viable involucrar a los estudiantes con la problemática de sus lugares de procedencia y orientarlos a futuro: el profesionista 
formado por la universidad intercultural debe tener el compromiso con la región (en el caso ideal debe regresar a trabajar a su comunidad de origen) para participar activamente en su transformación positiva.

La Vinculación Comunitaria se aplica tanto en el área de la investigación como en la enseñanza. Cada universidad intercultural se compromete a generar procesos de desarrollo en las áreas rurales y/o indígenas de la región de impacto respectiva, a través de dos estrategias. Primero, mediante la realización de proyectos de investigación y de desarrollo gestionados por los académicos, en los cuales generalmente participan también los alumnos. Segundo, mediante el desempeño de los estudiantes, quienes adquieren la experiencia necesaria para su futura profesión a través de las materias específicas de Vinculación Comunitaria (eje transversal de cada licenciatura) que implican prácticas de campo.

\section{Aspectos metodológicos}

La Universidad Intercultural del Estado de México (UIEM) en la que se realizó la presente investigación es la primera universidad intercultural fundada en México (se estableció en 2004). Fue seleccionada porque cuenta con una trayectoria suficientemente larga para suponer que haya reunido una abundante experiencia en el aspecto práctico de la Vinculación Comunitaria dentro de su región de impacto. La estrategia metodológica fue acceder a dicha experiencia a través de los testimonios personales de un grupo de diez egresados, uno de los ex-rectores y dos trabajadores académicos encargados de las prácticas de la Vinculación Comunitaria. Dichos testimonios se reunieron mediante la técnica de entrevista cualitativa y fueron complementados por la observación no participante dentro del ámbito de la UIEM y la revisión bibliográfica inicial.

Puesto que la intención fue estudiar la percepción que se tiene por parte de los participantes del modelo, se propuso desarrollar un trabajo de tipo cualitativo que diera cabida a la recolección de la voz y opinión de los entrevistados para evidenciar los procesos de la UIEM desde una gama de los actores internos.

En el caso de los profesionistas egresados, el grupo de informantes que más nos concierne por ser los receptores y practicantes de la Vinculación Comunitaria, 9 de 10 son oriundos de la zona noroccidental del Estado de México y 1 migró a esta zona desde Álamo, Veracruz, a edad temprana. Sus localidades de procedencia son: San Felipe del Progreso cabecera municipal (2), Emilio Portes Gil, comunidad en el municipio de San Felipe del Progreso (1), Atlacomulco, cabecera municipal (1), Rincón de la Candelaria, comunidad en el municipio de Atlacomulco (1), San Pedro de los Baños, comunidad en el municipio de Ixtlahuaca (1), Jocotitlán, cabecera municipal (1), San José del Rincón, cabecera municipal (1) y Santa María Nativitas, comunidad en el municipio de San José del Rincón (1).

En las comunidades de Emilio Portes Gil y San Pedro de los Baños, la población indígena es mayoritaria, en San Felipe del Progreso se trata aproximadamente de una quinta parte de la población, y en el resto de las localidades está debajo del 10\%; sin embargo, en todos los casos de trata de lugares con presencia indígena y de tipo más rural que urbano (con la excepción de la ciudad de Atlacomulco). De acuerdo con estos datos, 7 egresados entrevistados proceden de familias hablantes de la lengua mazahua (6) u otomí (1), aunque estos idiomas ya no sean la primera lengua de los informantes, y 3 son hablantes del castellano que provienen de familias mestizas. 


\section{Relación entre la universidad y las comunidades locales}

Como se adelantó en los párrafos introductorios, el punto de partida para entender cómo se relaciona la UIEM con las comunidades regionales (rurales y/o indígenas) es reconocer a la Universidad en su posición de proveedor de los servicios educativos y a las comunidades como unidades socioculturales y territoriales que son receptoras de esta política pública del estado mexicano. Es importante resaltar este dato porque el lenguaje utilizado en la teoría educativa de la universidad intercultural se empeña en ocupar una multitud de conceptos (interculturalidad, diálogo de saberes, etc.) que causan la impresión de que la universidad intercultural es una obra intercultural colectiva (indígena y no indígena) cuando en realidad el modelo educativo no fue concebido, ni está siendo gestionado mediante una toma de decisiones en conjunto con los pueblos originarios mexicanos.

Uno de los pocos comentarios al respecto fue hecho por Schmelkes, exdirectora de la CGEIB, la que declaró que la participación indígena en la construcción de las universidades interculturales había sido mínima (Schmelkes, 2008, pág. 336). Esto quiere decir que el modelo educativo establece desde el inicio una jerarquía entre cada universidad intercultural (ente activo: gestor) y las comunidades presentes en la región respectiva (entes pasivos: atendidos). De tal modo que en la UIEM existe personal académico y administrativo integrado tanto por los trabajadores "mestizos" hispanohablantes como por los de origen indígena y hablantes de lenguas originarias, pero en ambos casos su presencia se limita a los profesionistas con grados académicos, formados dentro del sistema educativo dominante. En cambio, la participación e influencia axiológica de las voces comunitarias indígenas, por ejemplo, las autoridades tradicionales o los ancianos, es marginal y no está en posición para tocar la esencia del modelo educativo.

Lo anterior puede comprobarse tanto al analizar los discursos oficiales, como observando directamente los acontecimientos al interior de la UIEM o revisando la bibliografía especializada (véase por ejemplo Warnholtz, 2013). Según la opinión detectada entre los alumnos de la UIEM por Molina (2012), no existe un vínculo suficiente entre las autoridades universitarias y las comunitarias, a las que se invita sólo para mostrarlas en las ceremonias públicas con su vestimenta tradicional, logrando un efecto folklórico colorido. Al respecto, Felipe González Ortiz, el primer rector de la UIEM, afirma lo siguiente:

La Universidad debe dirigir sus esfuerzos en el mediano plazo para que esté en manos completamente de los pueblos originarios... (...) El logro más optimista sería que arribáramos a la generación de conocimientos con metodologías distintas y epistemologías propias, y que salieran del pensamiento y de los lenguajes de los pueblos originarios3.

De manera análoga, Stefano Claudio Sartorello (2007), antropólogo y entre 2007 y 2013 docente de otra institución del mismo modelo educativo, la Universidad Intercultural de Chiapas, considera que más que abrir las comunidades a la universidad intercultural hace falta abrir la universidad intercultural a las comunidades, es decir, buscar una relación recíproca que supere la retórica del poder y sus discursos políticos conyunturales. Un ejemplo de tal "discurso conyuntural" puede ilustrarse en las palabras proporcionadas por el ex-rector de la UIEM, Francisco Monroy Gaytán:

${ }^{3}$ Entrevista a Felipe González Ortiz, en Celote (2013, pág. 158). 
(La vinculación comunitaria) es algo que ya no sé cómo empezó, pero es muy fuerte la vinculación, no sé si en un momento salimos, pero creo que en todos los trabajos de vinculación hubo la necesidad de trabajar, buscamos programas de financiamiento estatal, los vinculamos con ellos y se hizo una red. Y ahora ha ido creciendo y cada vez creo que se va consolidando más. (...) La aceptación ha sido mucha. Es mucho el entusiasmo porque en que debemos trabajar mucho es que ellos los habitantes de las comunidades) inicien y terminen proyectos. Me parece que está muy valorada entre las comunidades la Universidad (Intercultural), creo que tiene mucho impacto. Y la percepción, pues precisamente en el trabajo de unos investigadores geógrafos de la Universidad de Varsovia, lo primero que me dijeron después de un recorrido del trabajo de campo es que sentían que había muchísima aceptación en las diversas comunidades para la Universidad. Y así lo creo (entrevista a Francisco Monroy Gaytán, rector).

Esta visión optimista contrasta con un comentario más realista hecho por Gerardo Sánchez, comunicólogo de origen mazahua, durante un evento público en la UIEM, el cual ejemplifica un posible punto de vista de los actores comunitarios:

Ya tenemos una Universidad, la Universidad es muy bonita en la infraestructura y todo, pero desde mi punto de vista muy particular hace falta ahora meter el pensamiento y la palabra de los pueblos indígenas dentro de esta Universidad. (...) Tengo la esperanza de que ojalá los mazahuas se adueñen de esta Universidad, que el pensamiento realmente esté dentro de esta Universidad. He comentado, ojalá y la Universidad sea la semilla del pensamiento del pueblo mazahua, ojalá allá se disputen los grandes problemas que está viviendo el pueblo mazahua. Ojalá los chavos que están dentro de la Universidad regresen a sus comunidades para resolver los problemas, no para formar parte del problema sino para resolver los problemas 4 .

Esta muestra básica de la variedad de discursos pone en evidencia que la relación entre la Universidad Intercultural y las comunidades regionales es un tema complejo y controvertido. Para esclarecer este tema desde los actores directamente involucrados, adelante vamos a analizar los mecanismos propios de la Vinculación Comunitaria tal como fueron descritos por los académicos encargados de esta área y los egresados de la UIEM.

\section{Resultados. Las perspectivas sobre la realidad de la Vinculación Comunitaria desde dentro de la UIEM}

La problemática analizada se desdobla en los siguientes aspectos: con base en el eje llamado Vinculación Comunitaria, las estrategias empleadas a nivel de la UIEM hacia fuera y las aplicadas dentro de los procesos de enseñanza-aprendizaje de los alumnos.

En la UIEM podemos identificar dos proyectos clave. El primero se denomina Incubadora Social de Industrias Culturales, la que fue creada bajo el modelo de incubación de empresas

${ }^{4}$ Intervención de Gerardo Sánchez de la Radio Mazahua de Tuxpan durante la presentación del libro El nacimiento de la primera universidad intercultural de México (por Antolín Celote Preciado), 10 de marzo de 2014, UIEM, San Felipe del Progreso. 
apoyado por la Federación con la perspectiva de integrarse en los procesos de la Vinculación Comunitaria, impulsar economías solidarias y locales, y formar negocios sociales. La Incubadora trabaja con productores comunitarios (agricultores, artesanos, etc.), formando alianzas estratégicas con las diferentes cámaras (de Negocio, Libanesa, Nacional Restaurantera), secretarías (de Economía) y grupos (Wallmart), y consolidando los proyectos de negocio ante la Secretaría de Hacienda.

El segundo proyecto, el Tianguis Universitario, colabora directamente con la Incubadora, participando en los proyectos productivos sustentables en conjunto con los productores comunitarios, los que al inicio fueron contactados por los profesores de la Licenciatura en Desarrollo Sustentable. El Tianguis se ha llevado a cabo desde el año 2011 cada 15 días en los espacios de la UIEM, generando una ocasión para la venta, compra y trueque, donde además de los productores involucrados con la Incubadora participan curanderos, parteras, etc. También es una ocasión de enseñanza, ya que los alumnos entran en contacto con los artesanos y aprenden directamente de ellos.

Para conocer los modos de articulación de la UIEM con las comunidades mediante estas dos estrategias concretas, se entrevistó a dos académicos encargados, Alonso Reyes López y Micheline Dorcé Donnacien. Las entrevistas se centraron en esclarecer el protagonismo de la UIEM y de dichas comunidades en el proceso de diseño, gestión y realización de los proyectos de Vinculación Comunitaria.

En el caso de la Incubadora, los proyectos pueden surgir desde dos fuentes: a partir de las solicitudes de los actores internos (alumnos) o externos (personas de las comunidades 0 comunidades enteras). Según Alonso Reyes López, los primeros incubados fueron los estudiantes y sus padres quienes compartían sus ideas para aterrizar, tales como la crianza de borregos o conejos; también solicitaban apoyo para fortalecer negocios ya existentes. Un caso ilustrativo es el de Palmillas Plateros:

La comunidad se acercó. Los 23 productores de platería que ya estaban consolidados, a ellos se les estaba apoyando desde los años 70, pero nunca han tenido un crecimiento tal. Cuando llegan con nosotros, empezamos a formar este modelo de la triple hélice, donde buscamos financiamiento para ellos en la consolidación de organizaciones... Después hicimos con un centro de cultura una exposición del arte en plata y se les donó a través de empresas kilos de plata a los 23 productores para que hicieran su actividad y un espacio de exposición y venta (entrevista a Alonso Reyes López, académico).

Otra posibilidad es que un proyecto sea formulado por la UIEM y en forma de propuesta se lleve a las comunidades para ser considerado como opción por la gente local. En estos casos, la metodología del acercamiento consiste en sensibilizar a los interesados (alumnos, padres de familia, etc.) en cuestiones empresariales, asesorarlos para hacer "cuajar" sus ideas y posibilitar sus proyectos en la esfera institucional (instancias gubernamentales, organizaciones, cámaras de comercio). En este proceso entran diferentes limitantes, por ejemplo, el que las instituciones oficiales se apeguen estrictamente a la visión de desarrollo basada en fines de lucro, crecimiento económico y competitividad:

Cuando nosotros empezamos, no teníamos todo un planteamiento teórico y metodológico. Encontramos de entrada una institución que te dice que tienes que tener un plan de mercadotecnia, etcétera, entonces dijimos: "Bueno, la propuesta son pequeños productores, estamos con gente de un promedio de 1.5 o 2 hectáreas, producen para 
autosuficiencia, para autoconsumo." Y eso empezó a cuestionarse y hacía que un proyecto no podía pasar porque nos decían: "Es para que puedan vender sus cosas, no consumirlas ellos mismos" (entrevista a Micheline Dorcé Donnacien, académico).

El testimonio citado pone en evidencia la superposición de la perspectiva económica dominante sobre las visiones económicas locales. Estas últimas están influidas, aun en el altamente globalizado Estado de México, por las cosmovisiones indígenas y por la lógica de la convivencia comunitaria, en contraste con el individualismo moderno orientado hacia el crecimiento económico. Observaciones como ésta son relevantes para entender los alcances conceptuales del modelo educativo de la universidad intercultural, ante todo el concepto de la interculturalidad y el diálogo de saberes en posición de igualdad. Sin embargo, como puede observarse en la práctica cotidiana, existe cierta disparidad valorativa entre el conocimiento indígena tradicional y el científico moderno, siendo el último el primer inter pares.

Ahora bien, pasemos a las experiencias de los ex-alumnos con la Vinculación Comunitaria en la UIEM. En esta universidad, cada semestre se imparte una materia que debe preparar a los alumnos para el trabajo de campo con las comunidades de la región. La asignatura de Vinculación Comunitaria inicia desde el primer semestre con el objetivo de que los alumnos empiecen a buscar información y realizar investigación para reencontrarse con el pasado y presente de la comunidad, y así conseguir una especie de descubrimiento de lo propio (Celote, 2013, pág. 78; González, 2007, pág. 73). Los estudiantes eligen las localidades para vincularse suponiendo que, al ubicar las necesidades de la comunidad destino, irán madurando ideas que luego se materializarán en forma de un proyecto estudiantil o una tesis.

Los alumnos pueden realizar el trabajo de campo por grupos o equipos pequeños. Las salidas se organizan con la ayuda de un catedrático que tenga el conocimiento del lugar y pueda intermediar la entrada a la comunidad para los estudiantes. El lugar puede seleccionarse mediante la decisión colectiva de un equipo de trabajo, o también por la iniciativa de individuos concretos, ya que es posible que un alumno proponga su propia comunidad como destinataria del proyecto e incluso involucre a sus familiares. También se permite hacer trabajos totalmente individuales. Luego, con el respaldo de la Universidad, se realiza el acercamiento.

Para profundizar en esta problemática, conviene hacer hincapié que en las universidades interculturales estudian jóvenes con trasfondos vitales muy variados. Para empezar, hay que tocar la relevancia variante atribuida a la educación escolarizada a nivel superior por parte de la población regional, la cual es manifiesta en diferentes tipos de actitudes personales. En ciertos sectores comunitarios mexiquenses, los estudios no se consideran necesarios para adquirir bienes, debido a lo cual, más que la mitad de los padres de familia de los alumnos de la UIEM no reconocen la importancia de la formación universitaria (Silva y Rodríguez, 2012, pág. 87).

En adición, la experiencia de vida de los alumnos dista de ser homogénea. Algunos proceden de cabeceras municipales o ciudades, y por eso el ambiente comunitario puede quedar fuera de su experiencia directa y limitarse a conocimientos superficiales y a menudo estereotipados. Pero la mayoría del alumnado proviene de localidades marginadas y de familias con bajos recursos. Estos últimos aprovechan la ubicación accesible de las universidades interculturales, construidas en zonas rurales y de alta concentración étnica, y para este sector de población a menudo representan la única opción de realizar estudios universitarios. La UIEM se encuentra en la zona étnica mazahua y está situada en las afueras 
de la cabecera del municipio San Felipe del Progreso. Por eso, para una gran parte de sus alumnos, la comunidad es el ámbito de socialización primaria, lo cual es una característica que más los identifica con la universidad intercultural, según comprobó Molina (2012) precisamente en la UIEM.

Después de egresar, los vínculos entre el ex-alumno y la comunidad de origen pueden o disolverse, o mantenerse y seguir desarrollándose a través del desempeño laboral del nuevo profesionista. La primera situación está bien documentada sobre todo entre los jóvenes de raíces indígenas, egresados de las universidades convencionales, quienes suelen sumarse al mercado formal de tipo urbano, fuera de sus lugares de origen. En las comunidades rurales, el hecho de que un joven regrese a su lugar de origen bien puede ser visto como una señal de fracaso, sobre todo si no completa sus estudios. Las universidades interculturales procuran revertir esta creencia al formar a los alumnos para el involucramiento consciente con sus pueblos.

Revisemos ahora los testimonios particulares de estos entrevistados acerca de la Vinculación Comunitaria transcurrida durante sus estudios en la UIEM. La experiencia particular de cada egresado tiene que ver más que nada con su involucramiento anterior con los ámbitos comunitarios. Su percepción de la experiencia con el trabajo de campo va desde las vivencias no problemáticas con la comunidad hasta situaciones abiertamente conflictivas. La descripción detallada de las experiencias respectivas puede consultarse en el Cuadro 1:

\begin{tabular}{|c|c|c|c|}
\hline $\begin{array}{l}\text { Trasfondo familiar } \\
\text { lingüístico-cultural } \\
\text { del egresado }\end{array}$ & $\begin{array}{l}\text { Comunidad(es) } \\
\text { donde se realizó la } \\
\text { vinculación }\end{array}$ & $\begin{array}{l}\text { Resumen de la experiencia } \\
\text { personal }\end{array}$ & $\begin{array}{l}\text { Segui- } \\
\text { miento al } \\
\text { proyecto }\end{array}$ \\
\hline $\begin{array}{l}\text { 1. Ismael. } \\
\text { Hispanohablante } \\
\text { mestizo }\end{array}$ & $\begin{array}{l}\text { Rincón de la } \\
\text { Candelaria, } \\
\text { Atlacomulco }\end{array}$ & $\begin{array}{l}\text { Diagnóstico de } 3 \text { meses durante el } 2^{\circ} \\
\text { semestre de la licenciatura. Acogida } \\
\text { positiva en la comunidad debido al } \\
\text { permiso previo de la delegación. Los } \\
\text { habitantes locales no estaban } \\
\text { familiarizados con la UIEM, esperaban } \\
\text { recibir retribución por su colaboración } \\
\text { en el proyecto, había necesidad de } \\
\text { manejar situaciones delicadas. }\end{array}$ & No \\
\hline $\begin{array}{l}\text { 2. Yeni. } \\
\text { Hispanohablante } \\
\text { mestizo }\end{array}$ & $\begin{array}{l}\text { Emilio Portes Gil, San } \\
\text { Felipe del Progreso }\end{array}$ & $\begin{array}{l}\text { Investigación sobre las religiones y su } \\
\text { posición en la comunidad mediante la } \\
\text { aplicación de entrevistas. Acogida } \\
\text { positiva debido a la presencia de una } \\
\text { integrante del equipo originaria de la } \\
\text { comunidad, la familiaridad de su padre } \\
\text { en la localidad y el conocimiento previo } \\
\text { de la UIEM por parte de los habitantes } \\
\text { locales. }\end{array}$ & No \\
\hline $\begin{array}{l}\text { 3. Jeri. } \\
\text { Hispanohablante } \\
\text { mestizo }\end{array}$ & $\begin{array}{l}\text { Palmillas, San Felipe } \\
\text { del Progreso }\end{array}$ & $\begin{array}{l}\text { Investigación durante el } 1^{\mathrm{r}} \text { semestre de } \\
\text { la licenciatura sobre el contexto social de } \\
\text { las personas practicantes de la medicina } \\
\text { tradicional. Acogida problemática, ya } \\
\text { que el profesor de la materia no había } \\
\text { facilitado el contacto con la comunidad a } \\
\text { detrimento de la calidad del trabajo } \\
\text { realizado. Preocupación de los } \\
\text { integrantes del equipo por la posible } \\
\text { apropiación del trabajo por parte del } \\
\text { profesor llevó a la entrega del producto } \\
\text { final directamente a la delegación de la } \\
\text { comunidad estudiada. La opinión de que } \\
\text { la mayoría de los alumnos de la UIEM no }\end{array}$ & No \\
\hline
\end{tabular}


4. Edén.

Mazahuahablante
Jiquipilco

muestran interés por compartir el fruto de la investigación con las comunidades. Investigación de 15 días sobre las costumbres antes practicadas en la comunidad. Acogida final positiva tras un periodo de desconfianza y agresiones. La opinión de que la UIEM podría realizar excelentes proyectos si no faltara apoyo institucional.

\begin{tabular}{ll}
\hline 5. Norma. & San Juan Xalpa, \\
Mazahuahablante & Iztapalapa
\end{tabular}
Investigación durante el $1^{\mathrm{r}}$ semestre en No la materia Vinculación con la Comunidad. No hubo acuerdos claros sobre el trabajo realizado. Durante el resto de la carrera las salidas fueron escasas. La opinión de que la Vinculación Comunitaria era más practicada por los alumnos de la carrera Desarrollo Sustentable.

\begin{tabular}{ll}
\hline 6. Rosario. & San Juan Xalpa, San \\
Mazahuahablante & Felipe del Progreso
\end{tabular}

Investigación durante el $1^{\mathrm{r}}$ semestre en Mazahuahablante Felipe del Progreso la materia Vinculación con la Comunidad con la finalidad de recolectar los conocimientos tradicionales sobre las plantas medicinales. Acogida positiva, agrado de los habitantes ante el interés de los jóvenes por la medicina tradicional.

\begin{tabular}{ll}
\hline 7. Gaba. & San Miguel \\
Mazahuahablante & Tenochtitlán, San \\
& Felipe del Progreso
\end{tabular}

Investigación durante el $1^{\mathrm{r}}$ semestre, No recolección de datos sobre una leyenda local. El trabajo se realizó hacia al final del semestre y escaseó el tiempo. Acogida positiva debido a la actitud amistosa de los alumnos a pesar del desconocimiento de la lengua mazahua, extrañamiento de los habitantes ante el interés de los jóvenes por la lengua mazahua.

\begin{tabular}{ll}
\hline 8. Alma. & Comunidad de origen, \\
Mazahuahablante & varias comunidades \\
& del estado no \\
& especificadas
\end{tabular}

Cada semestre se realizaba un trabajo en una comunidad diferente (talleres, exposiciones, implementación de ecotecnias, proyectos ambientales, elaboración de una monografía sobre una comunidad que no se dio a conocer por inconclusa). Acogida positiva en la comunidad de origen, en las demás intermediaban los profesores. La UIEM era conocida en las comunidades adyacentes, en las remotas hacía falta una presentación.

\begin{tabular}{llll}
\hline $\begin{array}{l}\text { 9. Anayeli. } \\
\text { Mazahuahablante }\end{array}$ & $\begin{array}{l}\text { Providencia, San José } \\
\text { del Rincón }\end{array}$ & $\begin{array}{l}\text { Investigación sobre la historia de la No } \\
\text { comunidad mediante entrevistas con los } \\
\text { ancianos. Acogida positiva. }\end{array}$ \\
\hline $\begin{array}{ll}\text { 10. Griselda. } \\
\text { Otomíhablante }\end{array}$ & $\begin{array}{l}\text { Santa María Nativitas, } \\
\text { Mayorazgo, La } \\
\text { Concepción Chico, El } \\
\text { Tunal, San Miguel la } \\
\text { Labor en San Felipe } \\
\text { del Progreso (mun.) }\end{array}$ & $\begin{array}{l}\text { Domunentación historiográfica de la No } \\
\text { dirigida a los compañeros de clase. } \\
\text { Investigaciones posteriores en varias } \\
\text { comunidades del municipio (encuestas } \\
\text { sobre la situación social, cultural, } \\
\text { económica y política, tesis de grado). }\end{array}$ \\
& $\begin{array}{l}\text { Acogida tanto positiva como negativa, } \\
\text { debido a las tensiones provocadas por } \\
\text { ciertos tipos de preguntas. }\end{array}$ \\
\hline
\end{tabular}

Cuadro 1. Experiencias personales de los egresados con la Vinculación Comunitaria en la UIEM 
En los testimonios se nota que al iniciarse la vinculación, hay que tomar en cuenta varias circunstancias: el hecho de que en algunos lugares se desconozca la UIEM y su misión, lo cual ralentiza la colaboración, se presenta la ocasional falta de apoyo por parte de algunos docentes, asimismo, el bajo interés y compromiso de algunos alumnos, y también se agrega la tensión producida por las actitudes negativas arraigadas en las comunidades, tales como la desconfianza hacia cualquier persona desconocida que no sea "paisana" (específicamente hacia los investigadores advenedizos) y la costumbre de recibir apoyos e incentivos:

La situación en las comunidades es un tanto delicada cuando se trata de introducirse, generalmente esperan recibir algo, algún apoyo. Mucha de esa situación, me da pena decirlo, pero se lo debemos a nuestros políticos, ya que ellos son los que engañan a la gente de la comunidad cuando están en algún periodo de elección y jamás vuelven a la comunidad (entrevista a Ismael, egresado mestizo).

Al principio, las personas te ven un poco extraño y a veces suelen mostrarse un tanto agresivas, pero con el trato durante la práctica las personas se van dando cuenta que tus intenciones no son malas, entonces acceden a tus preguntas (entrevista a Edén, egresado mazahua).

Desde la lógica de su formación intercultural, los alumnos de la UIEM supuestamente deben estar equipados con ciertas habilidades (conocimiento de las lenguas originarias, metodología de diálogo intercultural, etc.) que les permitan entablar relaciones funcionales con una comunidad. Una de las estrategias básicas para establecer confianza es integrar en el equipo de trabajo a una persona conocedora del lugar, preferentemente oriunda y hablante de la lengua indígena local. Cuando el acercamiento a la comunidad es exitoso, generalmente lleva a experiencias vivenciales amenas:

Fue fácil adentrarse a la comunidad, ya que como fue un trabajo en equipo tuve la fortuna que una de mis compañeras pertenecía a dicha comunidad y el padre de ella era muy conocido en la misma, así que la obtención de la información no tuvo mayor problema (entrevista a Yeni, egresada mestiza).

Las personas son amables, nos dejaban entrar a su casa para ver qué tipo de plantas tenían, nos compartían pequeñas hojas de las plantas para utilizarlas en casa, algunas personas incluso nos invitaban el desayuno, mientras nuestra plática se extendía. La gente mayor tiene muchas cosas que contar y es agradable escucharlos. (...) Algunas personas se sorprendían porque nos interesáramos en nuestras tradiciones, les alegraba que nos interesáramos por los remedios naturales cuando en ese tiempo la medicina alópata nos rebasaba en todos los sentidos (entrevista a Rosario, egresada mazahua).

Aunque la mayoría de los testimonios señala haber recibido una acogida amistosa durante sus prácticas de campo o haberla podido desarrollar en el transcurso del tiempo (8 de 10), se menciona también una serie de limitantes que no permitieron cumplir con el trabajo en su plenitud. Dichas limitantes tienen que ver tanto con la organización institucional como con las actitudes personales de los actores involucrados.

Respecto al papel de la Universidad, el primer tema que sobresale en los testimonios es la cantidad de tiempo a dedicar al trabajo de campo. En este contexto señalemos que según un estudio realizado en 2014 (Medina y Hernández, 2014, pág. 60), una tercera parte de los 
egresados de la UIEM opinó que no se había dado suficiente importancia a las prácticas profesionales de la Vinculación Comunitaria. Algunos testimonios de la presente investigación apoyan esta postura:

No recuerdo cómo fue en primer semestre, después de eso ya casi no lo hicimos, de hecho, los que salían a comunidad eran los de Desarrollo Sustentable. Llevamos Tronco Común, era como tu última oportunidad para que decidieras qué carrera querías estudiar, y llevamos una materia llamada Vinculación con la Comunidad, sí salíamos, pero ya en la carrera casi no salíamos (entrevista a Norma, egresada mazahua).

Este problema se evidencia en los proyectos inconclusos sin seguimiento. Esta situación fue señalada por la totalidad de los entrevistados y pone en manifiesto que en el caso de que la Universidad no proporcione condiciones para que los alumnos desarrollen sus trabajos de manera continua en una sola comunidad a lo largo de la carrera, se complicará asegurar un resultado final sistemático y sólido, tal como un proyecto funcional o una tesis de grado mediante la cual el conocimiento recogido pueda ser regresado a la comunidad que lo había proporcionado:

Luego ya no regresamos (a la comunidad). La escuela tiene poco de haberse creado, yo considero que, si existiera apoyo por parte de ella, se lograrían buenos proyectos, mejor a los que hacen los extranjeros (entrevista a Edén, egresado mazahua).

Creo que el enfoque de la Universidad está mal, muy mal, se iluminan la boca de que ayudamos a los indígenas cuando no es así. Hacen eventos a morir y ¿de qué sirve? Deberían hacer proyectos reales, empezar y terminar o dejarlos en seguimiento con los miembros de la comunidad, investigaciones que sirvan, que tengan resultados, que se bajen recursos y que no se pierdan en el camino, que se vea la ayuda comunitaria (entrevista a Anayeli, egresada mazahua).

También los mismos académicos a cargo de la Vinculación Comunitaria señalan que resulta difícil mantener funcionando un equipo de trabajo estudiantil en el transcurso de la licenciatura, sobre todo porque las primeras prácticas de la Vinculación Comunitaria empiezan en el primer semestre (el llamado Tronco Común que corresponde a la nivelación académica) cuando los alumnos aún no habían escogido la carrera y en los próximos semestres ya siguen sus líneas y dejan de coordinarse.

Yo tuve el caso de unos chicos hace dos años, que incluso la chica promovió luz para su comunidad, la querían nombrar regidora de su comunidad porque hizo en tan poco tiempo muchas cosas... Y el comisariado le propuso que se quedara, que hiciera más por su comunidad, pero esta chica dijo: "Oye, yo soy del primer semestre, déjame seguir avanzando y a ver qué más se pueda hacer." Pero no, ya cuando pasó un semestre se dieron otras materias y cada quien a su división, se desintegró ese seguimiento que yo tenía porque la chica era de otra división (entrevista a Alonso Reyes López, académico).

Aunado a esto, no todos los alumnos están motivados a abandonar sus zonas de confort. Sienten que estudiar no implica salir del salón de clase y ante la incomodidad del trabajo de campo procuran reducir las prácticas al mínimo o de plano evitarlas. Esto repercute sobre la calidad de la conducta ética en los alumnos. Éstos no asumen la solidaridad y compromiso 
con las comunidades de las que extraen la información y no comparten el producto final del trabajo con los que los habían apoyado en el proceso de investigación (autoridades, informantes, guías, etc.).

La problemática recién expuesta se complementa por la conducta profesional de los profesores respectivos. En teoría se supone que el docente debe asesorar los trabajos estudiantiles e intermediar sus primeros contactos con las comunidades seleccionadas, pero en la práctica, no todos los profesores velan sobre estos asuntos, lo cual perjudica la calidad del trabajo estudiantil:

La gran mayoría de la comunidad ni supo a qué íbamos y allí siento que los profesores nos debieron orientar para guiarnos en la forma de vincular a las personas que no tienen ese conocimiento. Ahora pienso que pudimos haber hecho publicidad para que la persona que entrevistamos impartiera cursos o diplomados o al menos solo clases para los interesados. En fin, ahora se me ocurren cosas que en ese momento no me pasaron por la mente. El trabajo lo hicimos en el primer semestre, por eso no teníamos ni la más mínima idea de qué podemos hacer. No sabíamos que el campo de acción es muy grande y que pudimos hacer mucho más. (...) Es como si los profesores de aquí creyeran que en un semestre tú ya sabes cómo vincularte con cualquier comunidad, ya que lo tienes bien aprendido. (...) El trabajo lo entregamos al delegado de la comunidad y a la persona que entrevistamos constantemente, a la que visitamos para integrar la investigación. Eso ya fue decisión nuestra y sobre todo ética nuestra porque los profesores no se aseguraban que este trabajo llegara hasta los implicados en la comunidad. Creo que a muchos compañeros no les interesa ni les preocupa esto, solo su calificación, esto agranda las dificultades para las generaciones siguientes (entrevista a Jeri, egresado mestizo).

Señalemos a modo de cierre que después de egresar, entre los jóvenes entrevistados en general se nota una actitud positiva hacia el trabajo de campo. Relacionando su experiencia con la Vinculación Comunitaria y la actividad laboral después de la carrera, podemos afirmar que la mitad de ellos (5) se han desempeñado en alguna ocasión en los ámbitos comunitarios en sus trabajos respectivos. Por su parte, los 5 egresados restantes que no han hecho este tipo de labor declaran que desearían hacerla o no se opondrían si les llegara una oportunidad de este tipo. Esto deja una evidencia favorable sobre los valores sociales e interculturales de los egresados de la UIEM, aunque, para formular conclusiones más categóricas, habría que realizar una investigación más detallada sobre este aspecto de la educación superior intercultural.

\section{Conclusión}

La UIEM se relaciona con las comunidades rurales y/o indígenas de la región de impacto a través de una serie de estrategias conocidas como la Vinculación con la Comunidad (uno de los ejes de la teoría educativa de la Universidad Intercultural), que consisten en la articulación de proyectos institucionales (incubación de negocios, comercio justo) y proyectos estudiantiles en el marco de los procesos formativos. Esta relación se manifiesta por un lado en la voluntad de la UIEM para abrirse a las ideas surgidas desde los alumnos y las comunidades y apoyar su realización, pero por otro lado, también en una actitud modernizante que da prioridad a las nociones del desarrollo económico (creación de negocios, divulgación del pensamiento empresarial en las comunidades) y prácticas 
antropológicas de "rescate" de los saberes ancestrales que aún se conservan en las comunidades y que desembocan en las descripciones más bien superficiales de la mitología, medicina tradicional, historia, lengua, etc. Asimismo, parece no haber suficiente retroalimentación y aprovechamiento de los trabajos realizados por los estudiantes en las comunidades investigadas, ya sea en términos de seguimiento a lo largo de la carrera o el compromiso ético con las comunidades colaboradoras. De allí que la interculturalidad y el diálogo de saberes como los conceptos clave del modelo educativo de la Universidad en la práctica resultan en una desigualdad de posiciones entre los actores involucrados en el proyecto intercultural. 


\section{Agradecimientos}

Dirigimos un agradecimiento a todos quienes colaboraron para dar sentido al proyecto de investigación, a quienes vertieron su opinión: autoridades, académicos y alumnos.

\section{Bibliografía}

Bastida Muñoz, M. C. y Albino Garduño, R. (2011). "Participación académica en la vinculación con la comunidad: el caso de la Licenciatura en Desarrollo Sustentable de la UIEM", en Gerardo Bocco, Pedro Urquijo y Antonio Vieyra (Coords.), Geografía y ambiente en América Latina, México, UNAM.

Casillas Muñoz, M. y Santini Villar, L. (2006). Universidad Intercultural: un modelo educativo. SEP, México.

Celote Preciado, A. (2013). El nacimiento de la primera universidad intercultural de México Cuando el sueño se hizo palabra. CGEIB, México.

Erdösová, Z. y Juárez Toledo, R. (2017). "Nociones de bienestar, desarrollo y Buen Vivir. Aproximaciones discursivas en las Universidades Interculturales de Chiapas y el Estado de México", en Autoctonía, Universidad Bernardo O’Higgins, vol. 1, núm. 2, pp. 209-223.

Erdösová, Z. (2015). "Rethinking the borders inside Latin America and the clash of social imaginaries: the role of the Intercultural Universities in the indigenous autonomy in Ecuador and Mexico", en Central European Journal of International and Security Studies, Metropolitan University of Prague, núm. 9, vol. 3, pp. 42-57. Praga

Erdösová, Z. (2013). La universidad intercultural latinoamericana como un fenómeno múltiple. Una aproximación desde la teoría de los modelos educativos ecuatoriano y mexicano. Cuadernos interculturales, 11(21), 59-84.

González Ortiz, F. (2007). Crítica de la interculturalidad: la construcción de un proceso en el marco de la modernización. Cuadernos interculturales, 5(9), 63-89.

Korsbaek, L. y Sámano Rentería, M. A. (2007). “El indigenismo en México. Antecedentes y actualidad”, en Ra Ximhai, núm. 1, vol. 3, pp. 195-224. México

Medina, F. y Hernández García, R. M. (2014). Programa de seguimiento de egresados. Informe cuantitativo. San Felipe del Progreso: IUEM.

Molina, F. N. (2012). Los jóvenes en las universidades interculturales: la experiencia de integración al sistema universitario en la Universidad Intercultural del Estado de México. Universidades, LXII(54), 4-19.

Sartorello, S. (2007). Conversaciones interculturales: L@s estudiantes de la primera generación de la UNICH hablan de su universidad. San Cristóbal de las Casas: Gaceta, UNACH.

Schmelkes, S. (2008). “Creación y desarrollo inicial de las universidades interculturales en México: problemas, oportunidades, retos", en Daniel Mato (Coord.), Diversidad cultural e interculturalidad en educación superior. Experiencias en América Latina, Caracas, IESALC/UNESCO.

Silva Laya, M. y Rodríguez, A. (2012). El primer año universitario entre jóvenes provenientes de sectores de pobreza: un asunto de equidad. México: ANUIES. 
Warnholtz Locht, M. (2013). "Universidades interculturales, sin reconocimiento del Estado", en Animal Político, 5 de abril de 2013, recuperado de http://www.animal politico.com/blogueros-codices-geek/2013/04/05/universidades-indigenas-sinreconocimiento-del-estado-primera-parte/\#axzz2QDA7M6mC. 


\section{Autor}

ZUZANA ERDÖSOVÁ es Doctora en Estudios Latinoamericanos por la Universidad Autónoma del Estado de México (2015, mención honorífica y presea Ignacio Manuel Altamirano) y Doctora en Lenguas Romances por la Universidad de Palacký en la República Checa (2012). Es Maestra y Licenciada en Humanidades por la misma máxima casa de estudios checa. Desde 2016 se desempeña como profesora investigadora de tiempo completo en el Centro de Investigación en Ciencias Sociales y Humanidades (CICSyH) de la UAEM.

Es ganadora de la XIVa edición del Premio Iberoamericano (República Checa, 2009), candidata a Investigadora Nacional (SNI) y cuenta con el perfil deseable PRODEP. Es líder del Cuerpo Académico "Sociedades y Culturas de América Latina" adscrito a la Secretaría de Educación Pública en México. Es autora y coautora de varias decenas de publicaciones científicas sobre temas relacionados con su línea de investigación "interculturalidad, educación y lenguaje en América Latina". Ha sido ponente en diversos foros científicos nacionales e internacionales y ha dirigido diferentes proyectos de investigación. Se ha desempeñado como traductora, intérprete y también promotora de la cooperación académica y cultural entre México y la República Checa. 